\title{
The Role of Mental Rotations in Primate-inspired Robot Navigation
}

\author{
Ronald C. Arkin \\ School of Interactive Computing \\ Georgia Institute of Technology, Atlanta, GA U.S.A. 30332
}

\begin{abstract}
The use of a primate's spatial ability of mental rotation to serve as a basis for robotic navigation has been almost entirely overlooked by the robotics community to date. In this paper, the role of this cognitive capacity is presented as an adjunct to existing robotic control systems, with the underlying approach being derived from studies of primate spatial cognition. Specifically, optical flow is used as a basis for transitory representations (snapshots) that are compared to an a priori visual goal to provide corrective course action for a robot when moving through the world. The underlying architecture and procedures are described.
\end{abstract}

\section{Introduction}

The capacity for performing mental rotations has believed to have been observed in multiple species and not solely humans and thus begs the question as to whether it might have value in the context of robotics. The question persists regarding just what evolutionary advantage is afforded by this spatial reasoning system, especially for non-human primates, who do not use map reading. In an ongoing three year project for the Office of Naval Research, we are investigating the role of mental rotation in navigation for autonomous robots, not as a replacement for existing methods but rather as a conjunct for certain situations. Using optic flow derived depth maps as quasi-instantaneous snapshots of the environment, a behavioral robotic architecture is enhanced with this semi-reactive navigational capability based upon primate models of optic flow and mental rotation. To support this work, a vectorial mathematical framework is developed, spanning the sensorimotor and cognitive spatial aspects of this approach. This paper provides the motivation, background, theoretical basis, computational models, and results to date for utilizing primateinspired mental rotation models as a basis for intelligent robotic navigation.

While considerable scientific study has been devoted to mental rotation from both a psychological and neuroscientific perspective in humans (e.g., [Shepard and Cooper 82, Yule 06, Georgopoulos and Pellizzer 95, Khooshabeh 00]), primates (e.g., [Vauclair et al 93, Hopkins et al 93, Kohler et al 05]), and other mammals (e.g., [Mauck and Dehnhardt 97]), little is actually known about the underlying representations and processes by which it occurs. There are two primary schools of thought regarding representation: one involving propositional assertions (e.g., [Pylyshyn 73, Anderson 78]) and the other using analog visual reasoning (e.g., [Khooshabeh and Hegarty 10]). Our current research (Fig. 1) focuses on visual representations directly derived from imagery, exploring a multiplicity of representational options, including basis optic flow fields [Roberts et al 09], standard optic flow (Fig. 2b) [Gibson 79, Farneback 03], depth maps acquired either directly from a Kinect sensor (Fig. 2c) or generated from the optic flow field, and spatial occupancy grids (Fig. 2d) (e.g., [Elfes 89]) where each of these different representations is mapped onto the motor control system underlying the navigational control of a mobile robot [Arkin 89]. 


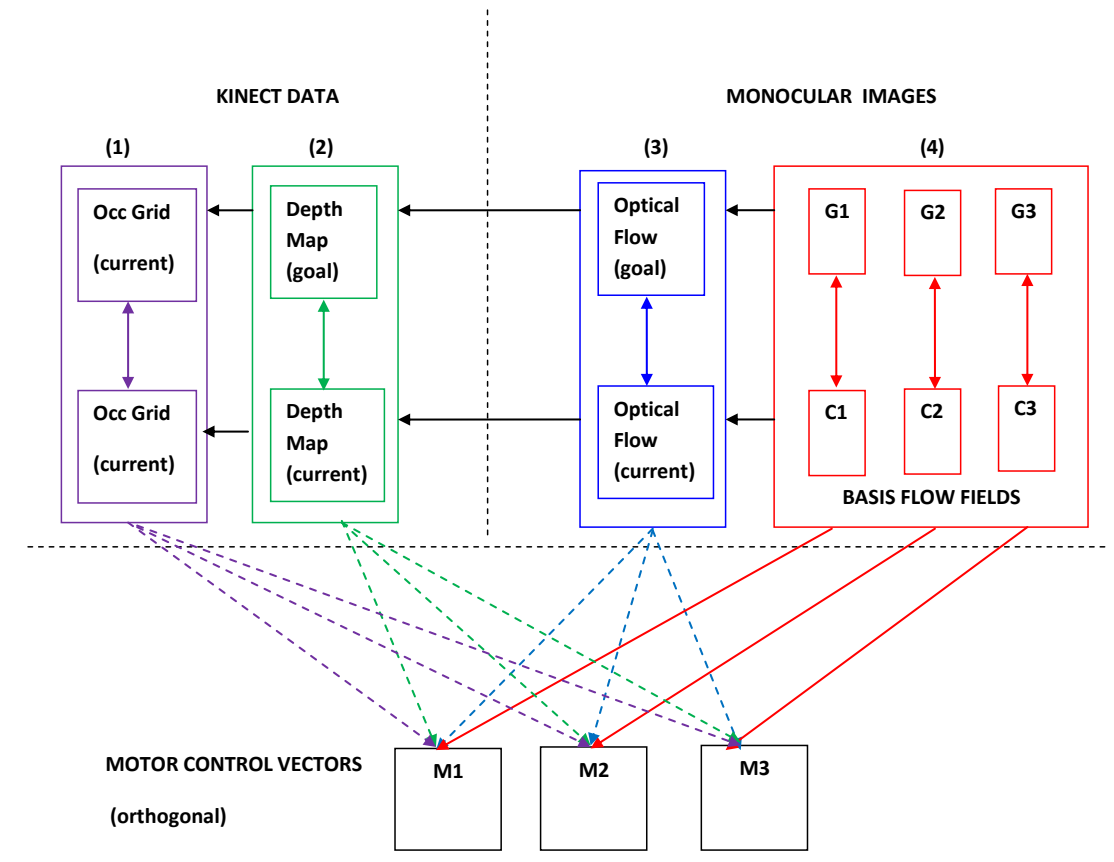

Figure 1: Multiple Representational Pathways to Motor Control

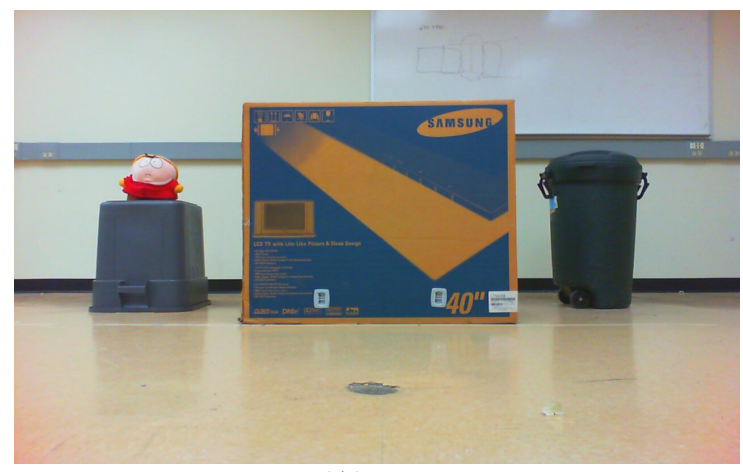

(A)

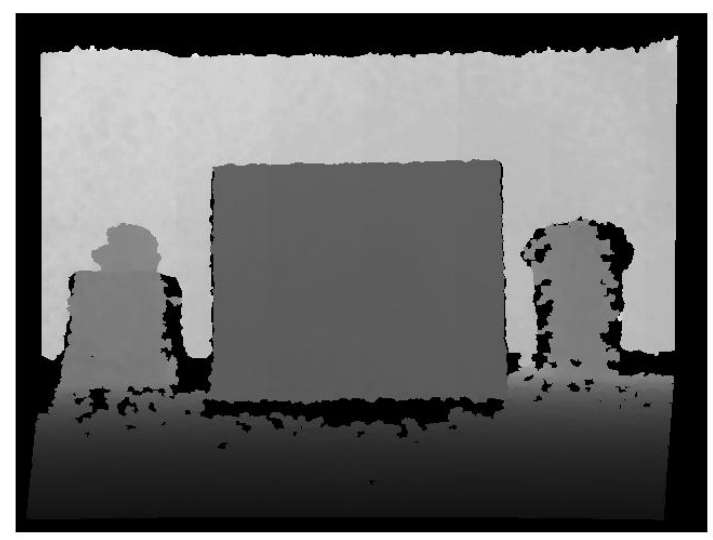

(C)

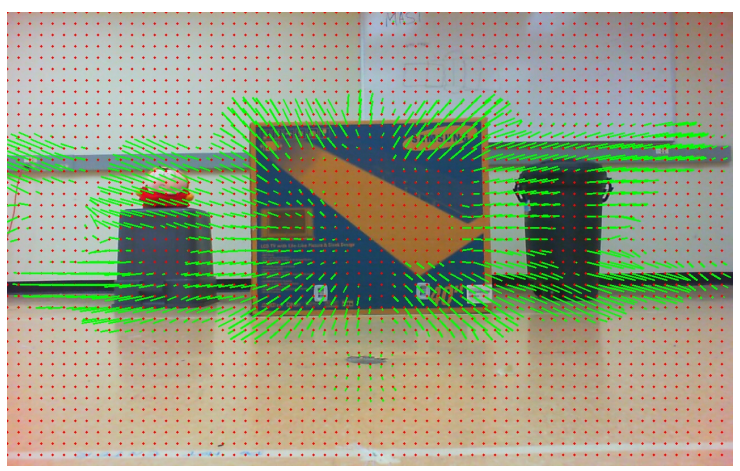

(B)

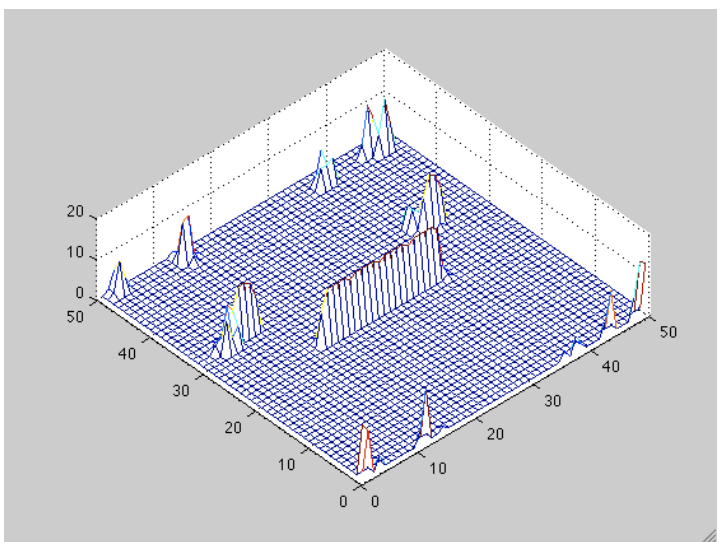

(D)

Figure 2: (A) Last image of optical flow sequence. (B) Optical Flow generated from sequence (C) Kinect depth map (D) Occupancy grid generated from depth map 


\section{A Role for Mental Rotation in Robot Navigation}

While mental rotation and its role in primate and human navigation has been studied to a degree by the spatial cognition and neuroscientific communities (e.g., [Shepard and Hurwitz 84, Wexler et al 94, Kozhevnikov et al 06]), relatively few studies have considered its role in robot navigation (e.g., [Taylor et al 08]). There already exist many excellent approaches for robotic navigational control that have developed over the past two decades (cf. [Arkin 98, Thrun et al 05]), so the question that confronts us is what, if anything, can adding the cognitive ability of mental rotation to a robot provide above and beyond these already existing capabilities. [Norman and Shallice 86] have shown that under certain circumstances willed cognitive mechanisms beyond automatic control are important, especially in situations involving troubleshooting, dangerous or difficult actions, or in novel or poorly learned situations. This can provide insight into opportunities to enhance already competent navigational methods.

The goal of our research is not necessarily to supplant existing robotic control strategies, but rather augment them with spatial capabilities derived from mental rotation research that facilitate proper action under these types of conditions. This requires two components: (1) recognition that such a situation warrants its use, i.e., the inadequacy of existing control methods is recognized through cognizant failure [Gat and Dorais 94]; and (2) the application of visual representations derived from optic flow that are used in process analogous to mental rotation, assisting in providing the necessary impetus for correct robot action at the right time. To accomplish the latter, a visual snapshot of a depth map or occupancy grid of a position near the spatial location of the robot's goal is stored a priori. A series of these serve as waypoints for the overall planned path which the robot must satisfy. Based on small incremental translational and rotational motions, local depth maps are created at the robot's present position while it is moving. These are local snapshots of the 3D world structure that the robot currently perceives (Fig. 3), cast in the same representational framework as the stored visual goal state.

Various correlation methods are being explored (e.g., [Hirshmuller 01, Lewis 95, Elfes 89]) to determine which might provide the best real-time correlation between local and goal depth maps (or even the direct optic flow representations) to provide intermittent yet frequent motor control updates for the robot.

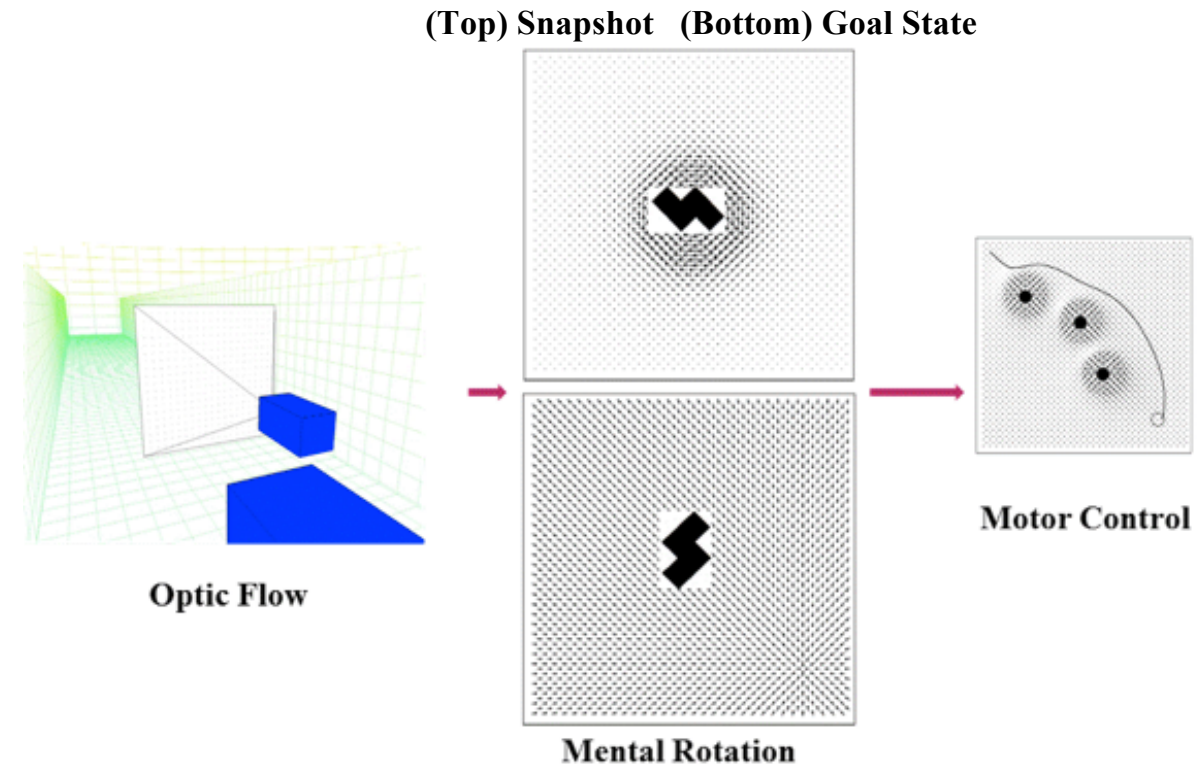

Figure 3: Flow of control from optical flow through spatial mental rotation to motor behavior 


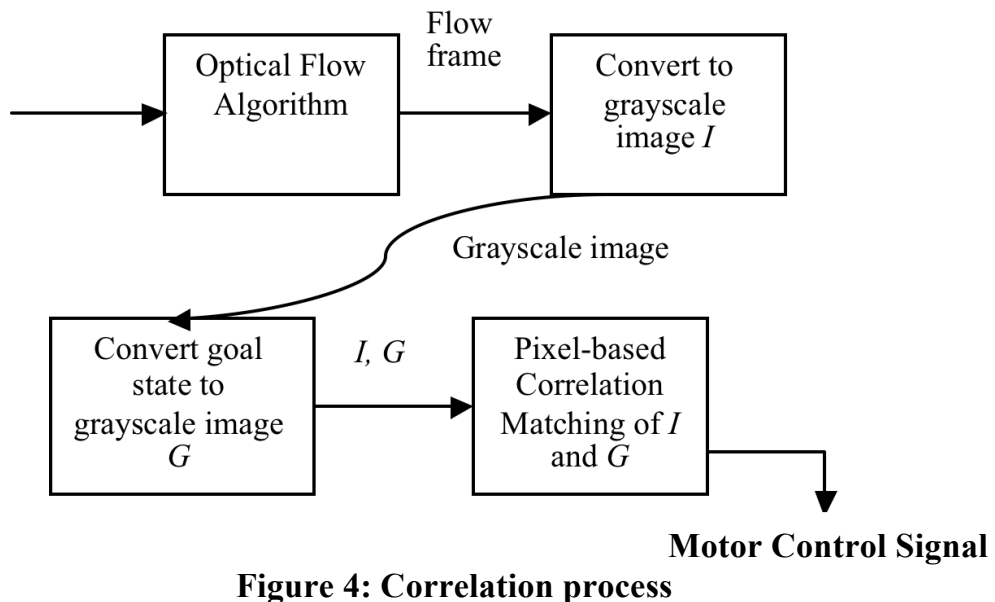

Another goal of this research is to find a spanning mathematical framework (interlingua) that can unite sensory processing, cognitive spatial manipulation, and motor control. Optical flow [Gibson 79, Farneback et al 03], a perceptual process for the derivation of structure from motion, has consistently used vectorial representations to represent the flow between monocular images, computing depth by correspondence and knowledge of the egomotion of the agent. Vectorial representations are omnipresent in motor control as well, both in biological systems (e.g., [Georgopoulos 86, Georgopoulos et al 86, Bizzi et al 91]) and robotic systems (e.g., [Arkin 89, Giszter et al 00]). While hybrid deliberative/reactive architectures provide a means for integrating higher level cognitive planning with purely reactive control [Arkin 98], a means for integrating mental rotation into the navigational process to date has been neglected in robotics. Casting the mental rotation as a motor process [Wexler 94] (fig. 3) may yet yield the missing link connecting cognition into the sensorimotor framework for robotics.

\section{Summary}

This research directly applies theories of mental rotation from the spatial cognition community to robot navigation with the goal of augmenting existing robotic navigational systems to cope with situations that might otherwise be confounding. Using optical flow as the basis for internal representation, a control regime is described which can potentially generate effective navigational solutions when confronted with novel, dangerous, confusing, or poorly learned, situations. This short paper outlines the architectural framework by which such augmentation can be provided based upon the correlation of visual depth maps representing transient positions and a desired goal state. In future work, this ongoing research will report on the efficacy of these various representations and correlations methods as the basis by which such navigational augmentation can be provided.

\section{Acknowledgments}

This research is supported by the Office of Naval Research under grant \#00014-11-1-0593. The author would like to thank Prof. Frank Dellaert from Georgia Tech and Prof. Kim Wallen from the Yerkes National Primate Research Center for their significant contributions to this project and to acknowledge the student team members for their important roles: Suresh Natesh, Joan Devassey, and Ivan Walker.

\section{References}

1. Anderson, J. R., “Arguments concerning representations for mental imagery”, Psychological Review, Vol. 85, pp. 249-277, 1978.

2. Arkin, R.C., "Motor Schema-Based Mobile Robot Navigation", International Journal of Robotics Research, Vol. 8, No. 4, pp. 92-112, August 1989. 
3. Arkin, R.C., Behavior-based Robotics, MIT Press, 1998.

4. Bizzi, E., Mussa-ivaldi, F., and Giszter, S., "Computations Underlying the Execution of Movement: A Biological Perspective", Science, Vol. 253, pp. 287-291, July 1991.

5. Derrode, S., "Robust and Efficient Fourier-Mellin Transform Approximations for Gray-Level Image Reconstruction and Complete Invariant Description", Computer Vision and Image Understanding 83, pp 57-78, 2001 .

6. Elfes, A., Occupancy Grids: A Stochastic Approach to Mobile Robot Perception and Navigation, Ph.D. Dissertation, CMU, 1989.

7. Farneback, G., "Two-frame Motion Estimation based on Polynomial Expansion", Lecture Notes in Computer Science, Springer, Vol. 2749, pp.363-370, 2003.

8. Gat, E. and Dorais, G., "Robot Navigation by Conditional Sequencing," 1994 IEEE International Conference on Robotics and Automation, San Diego, CA, May 8-13, 1994, vol. 2, pp. 1293-1299.

9. Georgopoulos, A., "On Reaching”, Annual Reviews Neuroscience, Vol. 9, pp. 147-70, 1986.

10. Georgopoulos, A. and Pellizzer, G., "The Mental and the Neural: Psychological and Neural Studies of Mental Rotation and Memory scanning”, Neuropsychologia, 33:1531-1547, 1995.

11. Georgopoulous, A., Schwartz, A., and Kettner, R., "Neuronal Population Coding of Movement Direction", Science, Vol. 233, pp. 1416-1419, 1986.

12. Gibson, J. J., The Ecological Approach to Visual Perception, Houghton Mifflin, Boston, 1979.

13. Giszter, S., Moxon, K., Rybak I. and Chapin, J., "A Neurobiological Perspective on Humanoid Robot Design", IEEE Intelligent Systems, pp. 64-69, July/August 2000.

14. H. Hirschmuller, "Improvements in real-time correlation-based stereo vision", Proceedings of the Stereo and Multi-Baseline Vision, pp 141-148, 2001.

15. Hopkins, W., Fagot, J., and Vauclair J., "Mirror-Image Matching and Mental Rotation Problem Solving by Baboons (Papio papio): Unilateral Input Enhances Performance," Journal of Experimental Psychology: General, Vol. 122, Issue 1, pp. 61-72, 1993.

16. Khooshabeh, P and Hegarty, M., "Representations of Shape during Mental Rotations", AAAI Spring Symposium Series, March 2010.

17. Khooshabeh, P., Is Mental Rotation Color Blind? Ph.D. Dissertation, Psychology Department, University of California, Santa Barbara, 1990.

18. Kohler, C., Hoffman, K., Dehnhardt, G., and Mauck, B., "Mental Rotation and Rotational Invariance in the Rhesus Monkey (Macaca mulatta)", Brain Behav. Evol., Vol. 66, pp. 258-166, 2005.

19. Kozhevnikov, M., Motes, M., Rasch, B., and Blajenkova, O., "Perspective-Taking versus Mental Rotation Transformations and How they Predict Spatial Navigation Performance”, Applied Cognitive Psychology, 20:397417, 2006.

20. J. P. Lewis, "Fast Normalized Cross-Correlation", Vision Interface, 1995.

21. Mauck B., and Dehnhardt, G., "Mental Rotations in a California Sea-Lion (Zalophus Californianus)", Journal of Experimental Biology, Vol. 200, pp. 1309-1316, 1997.

22. Norman, D. and Shallice, T., "Attention to Action: Willed and Automatic Control of Behavior", in Consciousness and Self-Regulation: Advances in Research and Theory, Vol. 4, (Eds. Davidson, M., et al), Plenum Press, pp. 1-17 1986.

23. Pylyshyn Z., "What the mind's eye tells the mind's brain: a critique of mental imagery", Psychological Bulletin 80: 1-24, 1973.

24. Roberts, R., Potthast, C., and Dellaert, F., "Learning general optical flow subspaces for egomotion estimation and detection of motion anomalies" Proc. IEEE Conf. on Computer Vision and Pattern Recognition, 2009.

25. Shepard, R and Cooper, L., Mental images and their transformations, Cambridge, MA: MIT Press, 1982.

26. Shepard, R. and Hurwitz, S, "Upward Direction, Mental Rotation, and Discrimination of Left and Right Turns in Maps", Cognition, Vol. 18, pp. 161-193, 1984.

27. Taylor, H.., Brunye, T., and Taylor, S., "Spatial Mental Representation: Implications for Navigation System Design", Reviews of Human Factors and Ergonomics, Volume 4, 2008.

28. Thrun, S., Burgard, W., and Fox, D., Probabilistic Robotics, MIT Press, 2005.

29. Vauclair, J., Fagot, J., an Hopkins, W., "Rotation of Mental Images in Baboons when the Visual Input is Directed to the Left Cerebral Hemisphere", Psychological Science, Vol. 4, No 2, pp. 99-103, March 1993.

30. Wexler, M., Kosslyn, S. and Berthoz, A., "Motor Processes in Mental Rotation”, Cognition, Vol. 66, pp. 77-94, Elsevier, 1994.

31. Yule, Peter. "A new spin on mental rotation." 1997, University of London. Accessed February 12, 2006 $<$ http://www.psyc.bbk.ac.uk/people/associates/pgy/notes/rotation/>. 